\title{
17. A multimodal perspective on data visualization
}

\author{
Tuomo Hiippala
}

\begin{abstract}
This chapter discusses the multimodality of data visualizations, that is, how they combine multiple modes of expression, such as written language, photographs, diagrammatic elements, and illustrations, in various printed and digital media. Because the medium in which a data visualization is presented determines the modes of expression available, the chapter shows how different media can be pulled apart for multimodal analysis. The proposed approach is illustrated by analysing static information graphics, non-interactive, and interactive dynamic data visualizations.
\end{abstract}

Keywords: Multimodality; Media; Data visualization; Information graphics; Interactive media

\section{Introduction}

The current interest in multimodality, or how multiple modes of communication cooperate and interact, has opened up new opportunities for theoretical reflection and practical application within several fields. Thus we find in linguistics increasingly widespread statements that language should be seen as just one form of communication among many other, equally important expressive resources; visual communication begins to consider aspects of language; art history takes in the moving image; humancomputer interaction design is extended to include tactile and gestural communication rather than just language, and so on. Research on data visualization, however, has not yet fully benefitted from the interdisciplinary perspective that defines most of the current research on multimodality. Previous research has established principles for visualizing information

Engebretsen, M. and H. Kennedy (eds.), Data Visualization in Society. Amsterdam: Amsterdam University Press, 2020 DOI 10.5117/9789463722902_CH17 
(Tufte, 1983, 1997), mapped the repertoires of visual expression (Bertin, 1981, 1983, 2001; Engelhardt, 2002) and explored the perception and reception of data visualizations (Holsanova, Holmberg, \& Holmqvist, 2009; Ware, 2012; de Haan et al., 2018).

Another stream of research has recently called for attention to how data visualizations may privilege certain perspectives or appear objective (Dick, 2015; Kennedy, Hill, Aiello, \& Allen, 2016) and identified inequalities in access to the kinds of literacies needed for making sense of data visualizations (D'Ignazio, 2017). These contributions have provided a much-needed critical perspective to complement the design- and reception-oriented approaches introduced above. At the same time, however, attempts to describe the multimodality of data visualizations have been relatively few (exceptions include Engebretsen \& Weber, 2017; Bateman, Wildfeuer, \& Hiippala, 2017), although Ledin and Machin (2018) have argued that any form of critical inquiry that targets contemporary forms of communication, such as data visualizations, must now be supported by a robust theory of multimodality. Ledin and Machin's (2018) call for increased support from theory resonates with the oft-cited quote from Halliday, who observed that:

A discourse analysis that is not based on grammar is not an analysis at all, but simply a running commentary on a text. (1994, p. xvi)

Although the notion of 'grammar' has been suggested as problematic for multimodal analysis, because it relies on strong assumptions about form, a property that visual modes of expression do not necessarily respect (Bateman, 2014a, 168), the need for a theory of multimodality that can reveal structural regularities and explain the choices made within specific modes of expression remains crucial for making systematic observations about multimodal discourse. Multimodal analyses are not only highly valuable on their own right for increasing our knowledge about multimodality as a phenomenon, but can also support critical perspectives on data visualizations by placing these analyses on a robust, multimodally-informed foundation.

A multimodal approach focuses on how meaningful combinations of written language, illustrations, photographs, diagrams, maps, layout, and other modes of expression emerge in data visualizations. How such combinations are supported multimodally and become interpretable across a wide range of different media remains a wide and open research question. For data visualization, answering these questions requires a theory capable of engaging with all forms of media in which data visualizations appear. 
In this chapter, I aim to show how the framework we proposed in Bateman et al. (2017) can be used as a foundation for analysing the multimodality of data visualizations. The proposed approach starts with a detailed account of the media in which data visualizations are presented, the modes of expression provided by the medium, and what kinds of engagement the combination of media and modes demands from those interacting with them. By doing so, I attempt to show how a comprehensive theory of multimodality can be used to identify the detail that is needed in critical inquiry (cf. Ledin \& Machin, 2018).

\section{Media and their canvases}

Data visualizations are used in different kinds of communicative situations across a wide range of media. They are presented on websites, printed on newspapers, shared on social media feeds, and projected on public displays, to name just a few examples (Lima, de Castro Andrade, Monat, \& Spinillo, 2014; Bounegru, Venturini, Gray, \& Jacomy, 2017; Amit-Danhi \& Shifman, 2018). For this reason, identifying the medium in which the data visualization is presented is a natural first step for their analysis, which has far-reaching consequences for a description of their multimodality. However, if media are characterized purely on the basis of their physical or technological characteristics, for instance, by setting up dichotomies such as 'print' or 'digital', we risk oversimplifying the medium in which data visualizations appear (see e.g. Elleström, 2010; Bateman, 2017).

In order to break down the abstract concept of 'media' (or medium) and prepare it for multimodal analysis, Bateman et al. (2017, pp. 86-87) adopt the notion of a canvas to describe any potential carrier of semiotic modes that may be taken up for interpretation. The notion of a canvas places very few demands on the underlying materiality — almost anything capable of carrying intentionally-produced signs will do. Thus a note scribbled on a napkin is just as interpretable as a daily menu written on a chalkboard, because the presence of semiotic modes signals that the canvas in question is offered up for interpretation. Multimodality research conceptualizes semiotic modes as socially-shaped resources for making and exchanging meanings, and just like semiotic modes, the canvases provided by a medium come to be by virtue of being embedded within a community of users (Bateman, 2011; Kress, 2014).

Bateman et al. (2017) propose that physical or technical media may be characterized as recognizable 'bundles' of canvases defined by patterns of 
production and consumption. To exemplify, the medium of news broadcast often allocates parts of the screen to news tickers, stock and weather information, and other overlays, in addition to the audiovisual broadcast (Tan, 2011). These parts of the medium differ from each other in terms of their characteristics: the notion of a canvas allows picking them out for description. From the consumer's perspective, identifying these canvases not only generates expectations about what kinds of communicative situations may take place on them, but also anticipates the semiotic modes most likely to be encountered in a particular communicative situation. In order to characterize the properties of a canvas, Bateman et al. (2017, p. 104) propose accounting for several material properties: space $(2 \mathrm{D}$ or $3 \mathrm{D})$, temporality (static or dynamic), transience (permanent or fleeting), and how the user is positioned with respect to the canvas (distanced observer or immersed participant).

Because some of these affordances are inherited from the materiality of the medium, this is also where differences begin to emerge between canvases. Nevertheless, all canvases that carry data visualizations must have an inherent spatial (2D) extent, which provides access to expressive resources provided by layout (Waller, 2012). First differences emerge within the temporal extent: spatial canvases without temporal extent are considered static, whereas their counterparts with a temporal extent can be characterized as dynamic. Dynamic canvases may be either immutable or mutable, which also determines their degree of interactivity (Weber, 2017, pp. 246-247). In most cases, these canvases are also designed, which in this context implies that the content (or underlying data) cannot be altered by the user.

For the multimodal analyst, being able to pick out canvases and their properties for closer analysis is crucial for making sense of how the underlying medium is used to support a data visualization. This is necessary for establishing differences between data visualizations presented on their own dedicated websites (see e.g. Zambrano \& Engelhardt, 2008; Bounegru et al., 2017) and those embedded in social media feeds, or for capturing the differences between information graphics in printed newspapers and their counterparts in digital media (see e.g. Lima et al., 2014). In both cases, the theoretical apparatus must be capable of taking on the complexity of the communicative situation in which a data visualization is mobilized, as opposed to hiding it from view. This is why the following section introduces an additional perspective, which attempts to capture the kinds of interaction demanded by canvases, shifting the attention from the production of canvases to their consumption. 


\section{Exploration and composition}

Dynamic data visualizations and static information graphics have been suggested to demand different kinds of engagement from their viewers (Lima et al., 2014; Weber, 2017). Bateman et al. (2017, p. 105) characterize this engagement in terms of ergodic work, redefining the concept originally developed by Aarseth (1997) for multimodality research. The concept of ergodic work seeks to characterize a communicative situation in terms of the effort required from those participating in the situation. More specifically, the concept emphasizes how participants co-construct the communicative situation they are interacting with/in and to what extent the participants may manipulate the situation (cf. Bucher \& Niemann, 2012). Because communicative situations can take place on canvases embedded within one another, different canvases may demand different forms of ergodic work.

As a form of ergodic work, engaging with interactive data visualizations may be broadly characterized as exploration (Bateman et al., 2017, p. 108). Exploration involves substantial ergodic work on behalf of the viewer, in the form of interacting with the visualization, for instance, by choosing which parts of the underlying data are rendered by manipulating the data visualization via an interface. The extent to which the visualization may be manipulated is naturally determined by its degree of interactivity (Weber, 2017, pp. 246-247). What remains beyond the user's reach, however, is the underlying data. In other words, the presentation of the data may be altered, but not the data themselves. For this reason, the communicative situation of engaging with an interactive data visualization may be characterized as ergodic yet immutable (Bateman et al., 2017, p. 108).

Another form of ergodic work required for interpreting data visualizations is that of composition, which requires the viewer to determine how the information presented on a $2 \mathrm{D}$ canvas is to be put together. The ergodic work of composition involves selective visual perception and interpretation, which may be revealed using methods such as eye-tracking, as Holsanova et al. (2009) and de Haan et al. (2018) have shown for data visualizations in printed and digital newspapers (Bateman et al., 2017, pp.107-108). It should be noted, however, that as forms of ergodic work, composition and exploration are not mutually exclusive. In fact, exploring a data visualization must necessarily involve ergodic work in the form of composition, as interpreting an interactive data visualization involves making sense of information rendered on the screen at a given point in time. These embedded forms of ergodic work emerge naturally from canvases embedded within each other (p. 109). 
To summarize, the concept of ergodic work draws attention to the different forms of engagement demanded by data visualizations. As the following analyses will show, differences in ergodic work may be traced back to the properties of the physical/technical medium in which the data visualization is realized.

\section{Three example analyses}

In this section, I demonstrate how the procedure set out in Bateman et al. (2017, p. 228) can be used to identify canvases in three different data visualizations, in order to lay a foundation for their multimodal analysis. All three examples discussed below are on the topic of sustainability, such as biological conservation, global warming, and marine pollution. The examples feature contributions from various semiotic modes in the form of written language, photography, diagrams, and graphic elements. For current purposes, I do not seek to pursue a detailed analysis of their structure and functions, but characterize them rather broadly. The same applies to any discourse relations that hold between them. In contrast, by focusing on the canvases I seek to provide the means for increased analytical control, laying a foundation for more detailed analyses.

\section{Static information graphics}

Figure 17.1 shows an information graphic produced by Graphic News, a London-based agency that produces news graphics for media outlets around the world. Whilst not a data visualization, this infographic contains visualized data alongside other elements, and as such the framework under discussion applies here. The information graphic combines several modes of expression - written language, photography, maps, two-dimensional illustrations, and diagrammatic elements - which are organized on several overlapping canvases. The wealth of semiotic modes present exemplifies why information graphics may be conceptualized as a composite semiotic mode, which provides the 'glue' necessary for joining together contributions from individual semiotic modes (Bateman et al., 2017, p. 289). This 'glue' may be traced back to a specific form of discourse semantics that supports the interpretation of such composite units, which uses the layout space to set up potential relations between elements that make up the composite unit (p. 264).

For this reason, interpreting information graphics requires ergodic work in the form of composition. The viewer must identify the semiotic modes, 


\section{Last male northern white rhino dies}

The world's last male northern white rhino has died after months of ill health, bringing the rhino subspecies a step closer to extinction

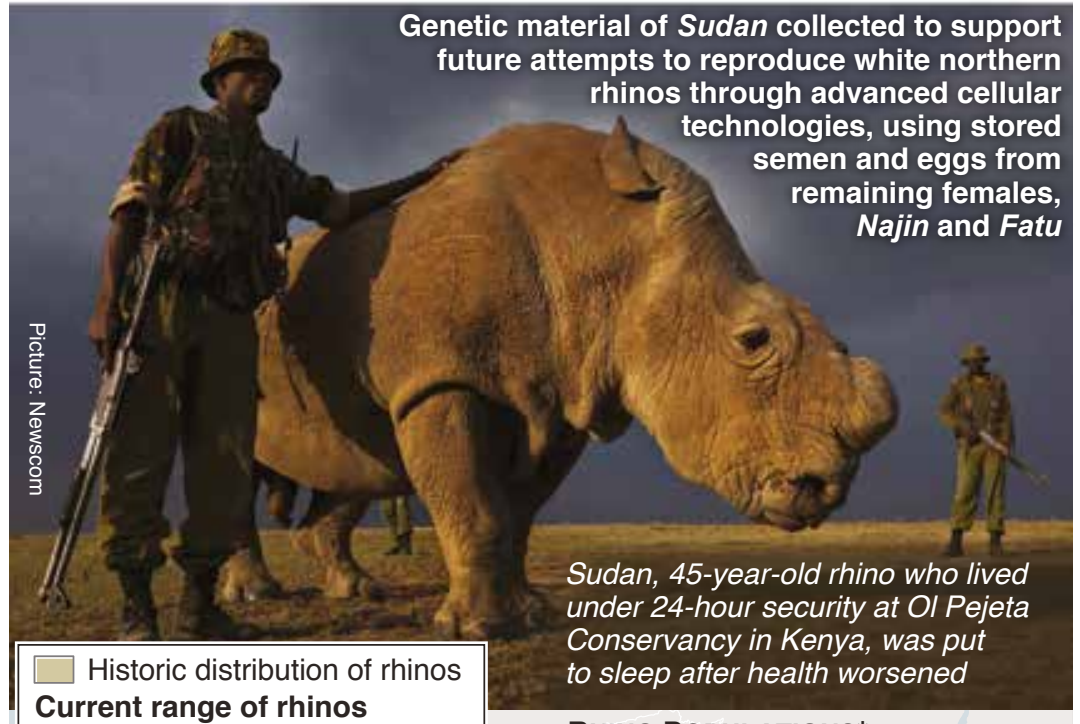

\section{Current range of rhinos}

White / Black

Indian, Javan and Sumatran

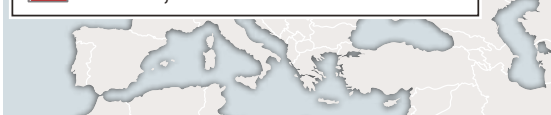

\section{RHINO POPULATIONS}

Indian rhino Status IUCN

Red List:

Vulnerable
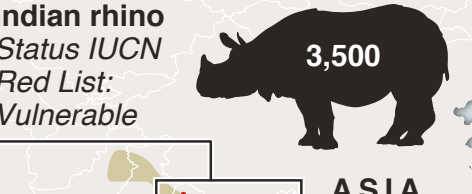

AFRIC A White rhino (Southern)

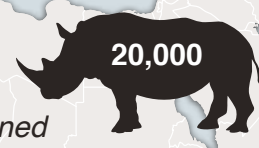

Near Threatened

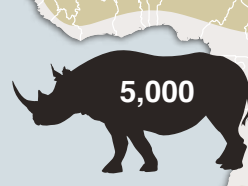

Black rhino

(Eastern, South-western, South-central) Critically Endangered

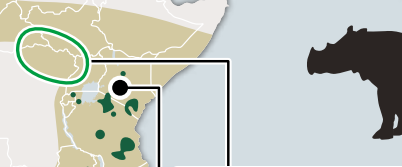

\section{Historic range} of northern white rhino

Sources: IUCN, Save the Rhino International

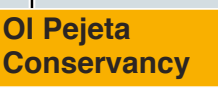

ASIA

${ }^{*}$ Figures at end of 2015

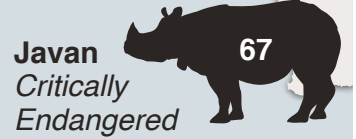

(C) GRAPHIC NEWS

Figure 17.1. A static information graphic reporting on the death of the last male northern white rhino. Produced by Graphic News. Copyright 2018 by Graphic News. Printed with permission. 
consider their specific contributions, and relate them to each other in the layout space. Resolving the discourse relations between written language and the photograph in the upper part of the graphic is a fairly trivial task, as written language is used to provide a headline, background information, and to identify the rhino in question. Making sense of the lower part, by contrast, may prove more challenging due to discourse relations that hold between multiple semiotic modes presented on several overlapping canvases, which are a common feature of information graphics (Bateman et al., 2017, p. 291).

The lower part of the visualization features a map that shows the current and historic geographical distributions of rhino populations. MacEachren (2004, p. 317) notes that maps use overlays to present complex phenomena in space and time, but required processes of attribution — assigning meaning to the overlays - are often dependent on other modes of expression. This process of attribution is exemplified in Figure 17.1 by the accompanying legend, which uses coloured graphic elements and written language to group together different species of rhino and establishes their current and historic spatial distributions. Laid out on top of the map is another canvas, which provides additional information on specific rhino populations using combinations of two-dimensional illustrations, written language, and diagrammatic elements, such as lines and containers. In addition, the diagrammatic mode is used to add information to the description in the upper part of the graphic by locating the Ol Pejeta Conservancy and the historic range of the northern white rhino.

These discourse relations, which are drawn between contributions from multiple semiotic modes and extend across the canvases, could be described in detail using various multimodal frameworks developed for this purpose. This level of description, however, is beyond the scope of this chapter (for a recent overview of this area, see Bateman, 2014b). In order to prepare for drawing comparisons between static and dynamic $2 \mathrm{D}$ canvases, it is worth noting how the static information graphic negotiates the limited layout space by using overlapping canvases. As the following examples will show, this limitation is largely absent from dynamic data visualizations, which can exploit material properties such as temporality and transience to increase the available layout space.

\section{Non-interactive dynamic data visualizations}

Figure 17.2 features four screenshots captured from a dynamic data visualization, which illustrates temperature anomalies by country between 1900 and 2016. Created by Antti Lipponen, a researcher at the Finnish Meteorological 


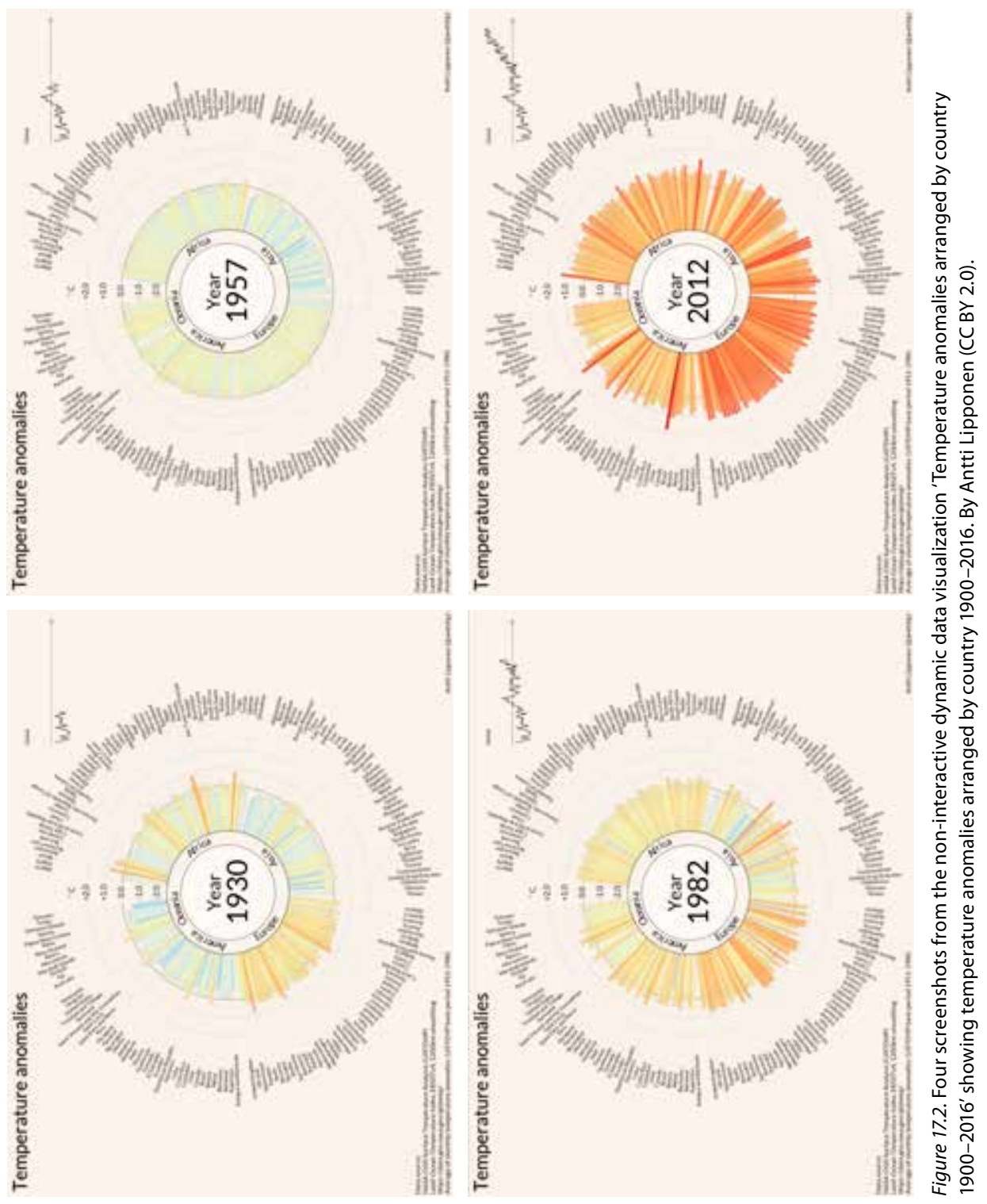

Institute, this $2 \mathrm{D}$ visualization is centred around a circular structure with the data-represented by coloured bars-and their respective labels laid out on concentric circles. The labels (countries) and bars (observations) are organized along the concentric circles according to geographical location. In addition, the top right-hand side of the visualization features a line graph which shows the global average temperature for each year, summarizing the individual observations presented using the circular bar plot.

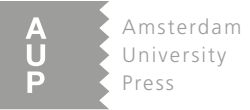


The visualization uses two semiotic resources provided by the diagrammatic mode to represent time series data, namely circular bar plots and line graphs (Tversky, 2017, p. 350). The visualization may be divided into three distinct canvases, which differ in terms of their temporality and transience. The first canvas, which carries the header, data sources, and author information, all positioned along the edges of the visualization, is static and permanent. The second canvas in the middle of the visualization is dynamic and fleeting in terms of temporality and transience, which enables the circular bar plot to be rendered again at each time step. Finally, the third canvas on the top right-hand side is also dynamic but permanent, which allows the line graph to be updated at each time step.

This difference in transience may be traced back to the diagrammatic resources and the kind of communicative work they are intended to do. Whereas the circular bar plot is used to represent changes among a large number of simultaneous observations, the line graph tracks a single observation over time to summarize the trend. The permanent canvas allows the line graph to use the two-dimensional layout space to keep all previous observations in view, which is something the circular bar plot cannot do: rendering each time step on the circular bar plot is simply not feasible due to limited layout space; the obvious solution is to distribute the representation over time, which is enabled by the fleeting canvas. Despite rapid changes, tracking changes on this canvas is facilitated by the way the human brain prioritizes the processing of colour and line length (Ware, 2012, pp. 154-155).

In terms of ergodic work, this visualization may be characterized as a dynamic data visualization, whose interpretation does not entail exploration, but constant composition. Exploration is not required, as animated graphics are not interactive, and consequently cannot support manipulation or navigation by the user (Weber, 2017, p. 247). Depending on whether the visualization is opened in a web browser or a media player application, initiating the temporal sequence may involve clicking a play button, but this interaction emerges from the medium in which the visualization is realized, not the visualization itself. Such low-level interactions are commonly used for imposing control over embedded dynamic canvases in digital media, and should not be confused with interactivity inherent to the data visualization (cf. Hiippala, 2017, pp. 424-425), which are taken up for discussion below.

\section{Interactive dynamic data visualizations}

The final example in Figure 17.3, The Seas of Plastic, is an interactive dynamic data visualization created by Dumpark, a design agency based in Wellington, 
New Zealand, which visualizes how plastic pollution accumulates into large circulating gyres in oceans. To do so, the visualization provides two distinct views - designated as map and source views, respectively_-which are both presented on their own canvases. These canvases may be rendered for viewing via the interface in the top-right corner of the embedding canvas, which remains constantly visible to the user.

In addition to the interface for exploring the visualization, additional levels of interactivity are introduced on the two canvases. The map view, shown in the upper part of Figure 17.3, features a $2 \mathrm{D}$ representation of a globe that may be freely rotated by clicking and dragging. A legend, positioned in the lower left-hand corner, is used to attribute meaning to the overlays rendered on the globe, which bears close resemblance to the discourse relations in the information graphic in Figure 17.3. The user may also select a specific gyre on the right-hand side interface, which rotates the globe into a position that shows the selected gyre. Selecting a source or a gyre in the source view highlights coloured bands that show the source or destination of plastic pollution. Multiple sources or gyres may also be selected simultaneously for drawing comparisons between them.

Together, multiple user interfaces on several canvases lend this data visualization a high degree of interactivity. According to Weber (2017, p. 247), this entails that the users are allowed to explore the visualization, interact with the data, and influence its representation, which corresponds closely with what Bateman et al. (2017, p. 108) characterize as ergodic work in the form of exploration. At the same time, the contributions from various semiotic modes and the discourse relations that hold between them closely resemble those found in static information graphics and non-interactive dynamic data visualizations: written language provides additional information on graphics, legends accompany cartographic representations, colour creates distinctions, etc., which must be decomposed and put back together for interpretation. In other words, making sense of the interactive data visualization requires ergodic work both in the form of exploration and composition, a feature which separates it from static information graphics and non-dynamic data visualizations.

\section{The need for exhaustive analyses}

Engebretsen and Weber (2017, p. 289) have recently argued that a multimodal account of data visualization must move beyond identifying which semiotic modes are used and for what purpose, and move towards a closer analysis of 


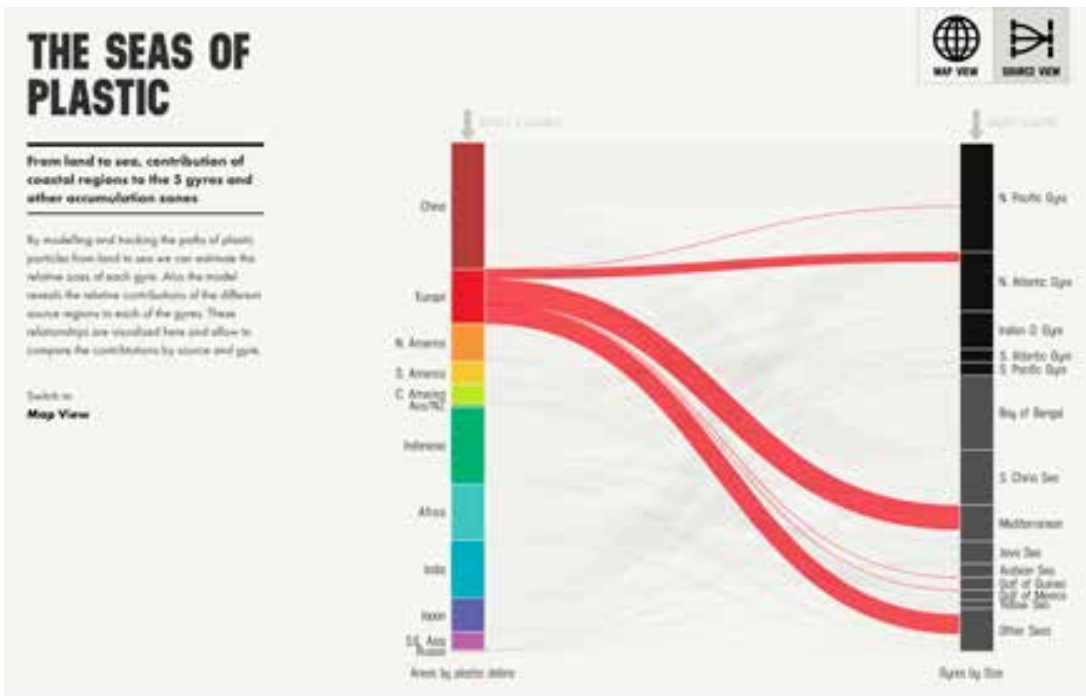

Figure 17.3. The Seas of Plastic, an interactive dynamic data visualization. Produced by Dumpark. Copyright 2018 by Dumpark. Printed with permission.

production and consumption, in order to pinpoint how meaning potential emerges. This argument is very similar to what Bateman et al. (2017, p. 221222) propose for multimodality research in general, underlining the need to pursue analyses in an exhaustive manner, which Ledin and Machin (2018) identify as a key component of any critical inquiry as well. Such analyses should involve (1) accounting for the communicative situations involved in engaging with a data visualization, (2) identifying the canvases on which these communicative situations take place, (3) identifying the semiotic modes mobilized on these canvases, and (4) the genres that shape the semiotic modes. This does not, however, necessarily entail full-blown analyses at each stage, but can also serve as a tool for limiting the scope of investigation.

That being said, identifying the canvases and describing their properties can be proposed as a first step towards a more comprehensive analysis of production processes. Canvases inherit affordances from the materiality of the medium that carries them, and they may be manipulated in different ways for different communicative purposes. What motivates the producers to manipulate these canvases and their material affordances can be revealed using ethnographic methods (Hiippala, 2016; Zha, 2017). For the examples discussed above, the properties of the canvases are visualized in Figure 17.4, in which they are marked as being either present (+) or absent (-).

The static information graphic (1) in Figure 17.4 illustrates how certain material properties of the medium are passed down to all canvases. The 

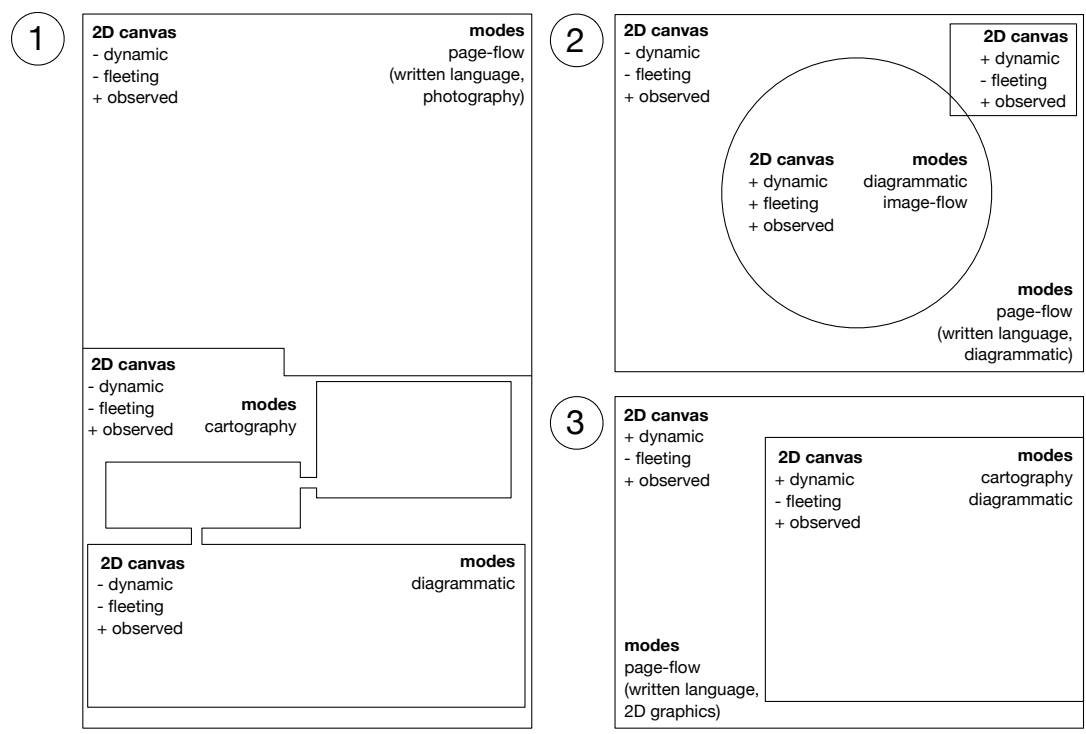

Figure 17.4 The decomposition of (1) static information graphics, (2) non-interactive data visualizations, and (3) interactive data visualizations into canvases. Illustration by T. Hiippala.

consequences are clear: a $2 \mathrm{D}$ medium without a material prerequisite for a temporal dimension can never be used to instantiate a dynamic canvas. This consequently rules out any semiotic modes that require this property. In contrast, the digital medium in which data visualizations (2) and (3) are realized affords controlling temporality and transience of any canvas, which also provides the foundation for interactivity. To summarize, the canvases and their properties determine which semiotic modes may appear on them, and thus their description should precede any in-depth description of the semiotic modes used and their contribution to the visualization at hand.

One contribution that emerges from mapping the canvases at play is the role of layout. Bateman (2008, p. 157) proposes the term page-flow for describing the semiotic mode responsible for setting up discourse relations in the layout space, which hold between contributions from distinct semiotic modes. The discourse semantics of page-flow are exemplified, for instance, by the relations that hold between the diagrammatic overlay and the underlying map in the static information graphic in Figure 17.1. The role of page-flow in organizing the spatial structure of $2 \mathrm{D}$ canvases is highlighted by indicating page-flow as the active semiotic mode in all visualizations in Figure 17.4. However, to what extent the discourse semantics of page-flow differ between data visualizations and entire page-based documents remains an open question for empirical research. 
To sum up, the widespread use of data visualizations makes their multimodal analysis challenging, given the wealth of communicative situations in which they appear. However, only a sufficiently developed theoretical apparatus, which is able to impose control on the communicative situation in which the visualizations appear, can advance our understanding of how the visualizations work. This will undoubtedly require an extensive programme of empirical research, which must involve specialists from various fields, given the need for exhaustive analyses that cover the whole range of phenomena from production to consumption (cf. Waller, 2012; Zha, 2017).

\section{Conclusion}

In this chapter, I have attempted to highlight how much state-of-the-art theories of multimodality can reveal about data visualizations even before venturing into in-depth descriptions of semiotic modes and the discourse relations that hold between their individual contributions to the data visualization under analysis. By drawing on the notion of canvas, recently introduced in Bateman et al. (2017), I have also sought to establish a foundation for further analysis by attending closely to the underlying properties of the medium, investigating their contribution to meaning-making, as called for by Ledin and Machin (2018). Such multimodally-informed insights could provide a basis for critical insights into the use of data visualizations in society, allowing them to be strongly rooted in well-informed analyses of multimodal discourse. Supporting these critical perspectives will also require continuous refinement of multimodal theories that are applicable to data visualizations, and given the rapid spread of data visualizations into all areas of society, these theories must undoubtedly be founded on empirical research.

\section{Acknowledgements}

I would like to thank Fiona Roberts of Graphic News, Antti Lipponen of the Finnish Meteorological Institute, and Timo Franz of Dumpark for giving the permission to reproduce the data visualizations discussed in this chapter. 


\section{References}

Aarseth, E. J. (1997). Cybertext: Perspectives on ergodic literature. Baltimore, MD: Johns Hopkins University Press.

Amit-Danhi, E. R., \& Shifman, L. (2018). Digital political infographics: A rhetorical palette of an emergent genre. New Media \& Society, 20(10), 3540-3559. https:// doi.org/10.1177/1461444817750565

Bateman, J. A. (2008). Multimodality and Genre: A foundation for the systematic analysis of multimodal documents. London: Palgrave Macmillan.

Bateman, J. A. (2011). The decomposability of semiotic modes. In: K. L. O'Halloran \& B. A. Smith (Eds.), Multimodal studies: Multiple approaches and domains. (pp. 17-38). London: Routledge.

Bateman, J. A. (2014a). Multimodal coherence research and its applications. In: H. Gruber \& G. Redeker (Eds.), The pragmatics of discourse coherence: Theories and applications. (pp. 145-177). Amsterdam: Benjamins.

Bateman, J. A. (2014b). Text and Image: A critical introduction to the visual/verbal divide. London and New York: Routledge.

Bateman, J. A. (2017). Triangulating transmediality: A multimodal semiotic framework relating media, modes and genres. Discourse, Context \& Media, 20, 160-174.

Bateman, J., Wildfeuer, J., \& Hiippala, T. (2017). Multimodality: Foundations, research and analysis - a problem-oriented introduction. Berlin: De Gruyter Mouton.

Bertin, J. (1981). Graphics and graphic information processing. Berlin: Walter de Gruyter. Bertin, J. (1983). Semiology of graphics: Diagrams, networks, maps (W. J. Berg, Trans.). Madison, WI: University of Wisconsin Press.

Bertin, J. (2001). Matrix theory of graphics. Information Design Journal, 1o(1), 5-19.

Bounegru, L., Venturini, T., Gray, J., \& Jacomy, M. (2017). Narrating networks: Exploring the affordances of networks as storytelling devices in journalism. Digital Journalism, 5(6), 699-730. https://doi.org/10.1080/21670811.2016.1186497

Bucher, H.-J., \& Niemann, P. (2012). Visualizing science: The reception of PowerPoint presentations. Visual Communication, 11(3), 283-306. https://doi. org/10.1177/1470357212446409

de Haan, Y., Kruikemeier, S., Lecheler, S., Smit, G., \& van der Nat, R. (2018). When does an infographic say more than a thousand words? Audience evaluations of news visualizations. Journalism Studies, 19(9), 1293-1312. https://doi.org/10.1 o8o/1461670X.2016.1267592

Dick, M. (2015). Just fancy that: An analysis of infographic propaganda in The Daily Express, 1956-1959. Journalism Studies, 16(2), 152-174. https://doi.org/10.1080/1 $461670 X .2013 .872415$ 
D'Ignazio, C. (2017). Creative data literacy: Bridging the gap between the data-haves and the data-have nots. Information Design Journal, 23(1), 6-18. https:/doi. org/10.1075/idj.23.1.03dig

Elleström, L. (2010). The modalities of media: A model for understanding intermedial relations. In: L. Elleström (Ed.), Media borders, multimodality and intermediality. (pp. 11-48). London: Palgrave.

Engebretsen, M., \& Weber, W. (2017). Graphic modes: The visual representation of data. In: C. Cotter \& D. Perrin (Eds.), The Routledge handbook of language and media. (pp. 277-295). New York and London: Routledge.

Engelhardt, Y. (2002). The language of graphics: A framework for the analysis of syntax and meaning in maps, charts and diagrams (Doctoral thesis). Institute for Logic, Language and Computation, University of Amsterdam.

Halliday, M. A. K. (1994). Introduction to functional grammar (2nd ed.). London: Arnold. Hiippala, T. (2016). Individual and collaborative semiotic work in document design. Hermes: Journal of Language and Communication in Business, 55, 45-59. https:// doi.org/10.7146/hjlcb.voi55.24288

Hiippala, T. (2017). The multimodality of digital longform journalism. Digital Journalism, 5(4), 420-442. https://doi.org/10.1080/21670811.2016.1169197

Holsanova, J., Holmberg, N., \& Holmqvist, K. (2009). Reading information graphics: The role of spatial contiguity and dual attentional guidance. Applied Cognitive Psychology, 23, 1215-1226. https://doi.org/10.1002/acp.1525

Kennedy, H., Hill, R. L., Aiello, G., \& Allen, W. (2016). The work that visualisation conventions do. Information, Communication and Society, 19(6), 715-735. https:// doi.org/10.1080/1369118X.2016.1153126

Kress, G. (2014). What is mode? In: C. Jewitt, C. (Ed.), The Routledge handbook of multimodal analysis (2nd ed.). (pp. 6o-75). London: Routledge.

Ledin, P., \& Machin, D. (2018). Doing critical discourse studies with multimodality: From metafunctions to materiality. Critical Discourse Studies. http://doi.org/10 $.1080 / 17405904.2018 .1468789$

Lima, R. C., de Castro Andrade, R., Monat, A. S., \& Spinillo, C. G. (2014). The relation between online and print information graphics for newspapers. In: A. Marcus (Ed.), Design, user experience, and usability: User experience design for everyday life applications and services. (pp. 184-194). New York: Springer.

MacEachren, A. M. (2004). How maps work: Representation, visualization and design. New York: Guilford.

Tan, S. (2011). Facts, opinions, and media spectacle: Exploring representations of business news on the internet. Discourse \& Communication, $5(2), 169-194$. https:// doi.org/10.1177/1750481311399511

Tufte, E. R. (1983). The visual display of quantitative information. Chesire, CT: Graphics Press. 
Tufte, E. R. (1997). Visual explanations: Images and quantities, evidence and narrative. Cheshire, CT: Graphics Press.

Tversky, B. (2017). Diagrams: Cognitive foundations for design. In: A. Black, P. Luna, O. Lund, \& S. Walker (Eds.), Information design: Research and practice. (pp. 349-36o). London: Routledge.

Waller, R. (2012). Graphic literacies for a digital age: The survival of layout. The Information Society, 28(4), 236-252. https://doi.org/10.108o/01972243.2012.6896o9 Ware, C. (2012). Information visualization: Perception for design (3rd ed.). Amsterdam: Elsevier.

Weber, W. (2017). Interactive information graphics: A framework for classifying a visual genre. In: A. Black, P. Luna, O. Lund, \& S. Walker (Eds.), Information design: Research and practice. (pp. 243-256. London: Routledge.

Zambrano, R. N., \& Engelhardt, Y. (2008). Diagrams for the masses: Raising public awareness - from Neurath to Gapminder and Google Earth. In: G. Stapleton, J. Howse, \& J. Lee (Eds.) Diagrams 20o8, volume 5223 of LNAI. (pp. 282-292). Springer, Berlin.

Zha, Y. (2017). Unpacking communication tensions in visual transmediation from print to digital papers. Communication Design, 5(1-2), 165-182. https://doi.org/1 $0.1080 / 20557132.2017 .1402501$

\section{About the author}

Tuomo Hiippala is Assistant Professor of English and Digital Humanities at the University of Helsinki, Finland. His major publications include The Structure of Multimodal Documents (2015, Routledge) and Multimodality: Foundations, Research and Analysis (2017, De Gruyter, with John A. Bateman and Janina Wildfeuer). 
A Amsterdam

U University

P Press 